\title{
ANÁLISE TERMO-ESTRUTURAL DE UMA VIGA EM SITUAÇÃO DE INCÊNDIO
}

\author{
BERNARDI, ANA PAULA \\ Engenheira Civil \\ Pontifícia Universidade Católica do Rio Grande do \\ Sul \\ Rio Grande do Sul; Brasil \\ anapaula.bernardii@hotmail.com \\ LAZZARI, PAULA MANICA \\ Professora Doutora \\ Universidade Federal do Rio Grande do Sul \\ Rio Grande do Sul; Brasil \\ p.manica.lazzari@gmail.com
}

\author{
LAZZARI, BRUNA MANICA \\ Professora Mestre / Doutoranda \\ Pontifícia Universidade Católica do Rio Grande do \\ Sul / Universidade Federal do Rio Grande do Sul \\ Rio Grande do Sul; Brasil \\ bruna.ml@gmail.com \\ FERREIRA, MATHEUS WANGLON \\ Doutorando \\ Programa de Pós Graduação em Engenharia Civil da \\ Universidade Federal do Rio Grande do Sul (PPGEC- \\ UFRGS) \\ Rio Grande do Sul; Brasil \\ matheuswanglon@gmail.com
}

\section{RESUMO}

Frequentemente, o material concreto armado é assunto de pesquisas e trabalhos acadêmicos, devido ao seu comportamento extremamente complexo e sua enorme importância na construção civil, sendo um dos materiais mais utilizados atualmente. Sabe-se que quando submetido a altas temperaturas, o concreto e o aço sofrem um decréscimo significativo nas suas propriedades resistentes. Além desta redução, o aquecimento do concreto acarreta outros efeitos nocivos à estrutura, como o efeito de spalling. Com o intuito de avaliar estas perdas de resistência, primeiramente realizou-se uma modelagem computacional com o software ANSYS, que funciona a partir do método dos elementos finitos, a fim de se obter a distribuição de temperatura em uma viga de concreto armado em situação de incêndio, o qual foi representado pela curva de incêndio padrão ISO 834 (2014). Após este passo, realizou-se a verificação analítica dos momentos últimos da viga antes e depois do incêndio evidenciando a fragilização da estrutura. Por fim, pode-se afirmar que o modelo proposto rendeu resultados satisfatórios.

Palavras-chave: análise térmica, incênio, elementos finitos, ANSYS, spalling

\begin{abstract}
Often, reinforced concrete material is subject of research and academic work because of its extremely complex behavior and its enormous importance in construction, being one of the most widely used materials nowadays. It is known when subjected to high temperatures, concrete and steel undergo a significant decrease in their mechanical properties. Besides this reduction, or heating of the concrete, other harmful effects to the structure, such as the spalling effect. In order to evaluate these resistances, first a computational modeling was performed throught software ANSYS, which works from the finite element method, in order to obtain the temperature distribution in a reinforced concrete beam in the fire situation, which was represented by the standard ISO 834 (2014) fire curve. After this step, an analytical verification of the last moments of the beam before and after the fire was carried out, showing the fragility of the structure Finally, it may be that the proposed model receives satisfactory results for the purpose.
\end{abstract}

Keywords: thermal analysis, fire, finite element, ANSYS, spalling.

\section{INTRODUÇÃO}

O material concreto armado é um constante alvo de pesquisas e de publicações acadêmicas, as quais exploram seus mais diversos aspectos relacionados ao cálculo estrutural e comportamento mecânico. Segundo Lazzari (2015), a justificativa para isto é a enorme importância que o material possui dentro da engenharia estrutural e também em função do seu comportamento ser extremamente complexo, quando submetido aos mais variados tipos de solicitações. 
Atualmente, o concreto armado pode ser empregado em todos os tipos de construção em função de suas diversas vantagens. Estas incluem, por exemplo, o fato do mesmo ser facilmente moldável, adaptando-se a qualquer tipo de forma, ser resistente ao fogo, às influências atmosféricas, ao desgaste mecânico, e, principalmente, por ser econômico, uma vez que a sua matéria prima (areia e brita) possui um custo relativamente mais baixo quando comparado com outros materiais (AURICH, 2001).

Entretanto, o material concreto armado, que é formado por barras de aço e uma matriz em concreto, quando submetido a altas temperaturas, pode sofrer um severo decréscimo na resistência à compressão do concreto e na tensão de escoamento do aço comum, além de afetar os valores do módulo de elasticidade do material. O processo de aquecimento da estrutura conduz, portanto, à redução da resistência dos materiais e da capacidade última dos elementos estruturais, além da ocorrência de esforços solicitantes decorrentes de alongamentos axiais restringidos ou de gradientes térmicos (CHUMBINHO, 2017).

Muito mais complexo do que uma redução de resistência, o aquecimento do material concreto acarreta na ocorrência do efeito de spalling, que se caracteriza pelo desplacamento de blocos de concreto da estrutura, os quais tornam a seção mais esbelta. Este é um efeito de difícil simulação, mas muito importante no estudo de estruturas em situação de incêdio.

De acordo com a NBR 15200 (ABNT, 2012), o objetivo da verificação de estruturas em situação de incêndio visa, basicamente, limitar os riscos de perdas de vidas humanas, assim como limitar o risco para a vizinhança, para sociedade e da propriedade exposta ao fogo. Para isto, a NBR 15200 (ABNT, 2012) estabelece critérios de projeto de estruturas de concreto em situação de incêndio, conforme Tempo Requerido de Resistência ao Fogo (TRRF), estabelecidos pela NBR 14432 (ABNT, 2001) para os diferentes tipos de estruturas e situações.

Dessa forma, este trabalho tem como objetivo principal estudar e avaliar o comportamento de uma viga em concreto armado quando exposta a temperaturas elevadas, como a curva de incêndio padrão ISO 834 (2014). Para isto, foram utilizados os parâmetros dos materiais e a geometria das vigas ensaiadas experimentalmente à flexão por Leonhardt e Walther (1962). O processamento de dados foi feito através do software ANSYS (Analysis Sistems Incorporated) versão estudante 2019R1, que é um programa computacional para análise de estruturas através da modelagem por elementos finitos. Vale ressaltar que o efeito spalling não foi considerado na modelagem computacional, devido a alta complexidade de simulação. A análise térmica adotada tem por base trabalhos percursores (Kumar e Kodur (2017); Ferreira (2019); Ferreira et al. (2019)) onde a metodologia foi validada através de comparações com resultados experimentais e analíticos.

\section{REFERENCIAL TEÓRICO}

\subsection{Software ANSYS}

As primeiras aplicações do método dos elementos finitos em estruturas de concreto armado datam de cerca de 30 anos atrás (AURICH, 2001). Atualmente, o método dos elementos finitos já se tornou uma ferramenta consagrada e mundialmente reconhecida, por sua capacidade de analisar estruturas muito complexas, como é o caso das estruturas em concreto armado, de uma maneira mais realista, simulando facilmente diferentes disposições geométricas, tipos de carregamentos e condições de contorno, através de um recurso computacional. O método considera a discretização de uma estrutura em um determinado número de elementos finitos, cujos comportamentos individuais são conhecidos (LAZZARI, 2015).

À medida que se refina esta malha, aumentando-se o número de elementos finitos, melhora-se também a precisão dos resultados do modelo discreto. Desta forma, a partir de um certo ponto, em que se trabalha com centenas ou milhares de elementos finitos, dados e resultados, só é viável utilizar o método através de programações automáticas ou sistemas computacionais de análises (SORIANO, 2003).

Além da precisão nos resultados, a utilização de um software de modelagem pelo método de elementos finitos para a realização de análises térmicas, possibilita a redução da quantidade de ensaios experimentais necessários, e, consequentemente, dos custos e do tempo dispendido neste processo. Porém, vale ressaltar que uma análise numérica não exclui a necessidade de realização de ensaios experimentais, uma vez que os resultados de ambos devem andar em conjunto. 
Diversos sistemas computacionais que utilizam o método dos elementos finitos podem ser encontrados no mercado atualmente. Entre eles pode-se destacar o software ANSYS, versão estudante 2019R1, utilizado nas mais diversas áreas da engenharia, o qual permite a realização de análises estáticas, dinâmicas e de fluidos, com materiais lineares e não lineares. O ANSYS dispõe de dois ambientes de trabalho, sendo o sistema workbench e o sistema APDL (ANSYS Parametric Desing Language), o qual permite que o usuário realize a modelagem através de uma programação, possibilitando a inclusão de comandos e todos os tipos de informações necessárias, tais como geometria e proprierdades do material, por meio de um arquivo de texto, com extensão .txt (KOCH, 2017).

No software a caraterização dos materiais da estrutura inicia a partir da escolha de elementos, os quais, assim como os comandos utilizados na modelagem, fazem parte da própria biblioteca do software. Neste caso os elementos escolhidos foram: SOLID 70, LINK 33 e SURF 152.

A modelagem do material concreto se deu a partir da utilização do elemento SOLID 70, um elemento tridimensional de oito nós e que possui capacidade de condução térmica. Já o elemento LINK 33 foi utilizado para a modelagem do aço, um elemento que, assim como o SOLID 70, é tridimensional e possui capacidade de condução térmica. As variáveis adotadas para a representação destes materiais no software foram: densidade, calor específico, emissividade e condutividade, sendo que as equações que determinam as propriedades do concreto podem ser encontradas no anexo $\mathrm{C}$ da NBR 15200 (ABNT, 2012) e as equações referente ao material aço se encontram na norma NBR 14323 (ABNT, 2013).

Para que a simulação de um incêndio seja a mais real possível, é necessário incluir nesta análise elementos de superfície sobre as faces da estrutura expostas ao fogo. A utilização de um elemento de superfície se faz necessária pois a temperatura que sai da fonte de calor não é a mesma temperatura que chega na estrutura, ou seja, a partir da utilização do elemento de superfície é possível considerar os efeitos oriundos da convecção e da radiação do material e através do balanço energético entre a fonte de calor e a superfície aquecida, pode-se determinar o quanto da energia emitida é realmente absorvida pela estrutura $\mathrm{O}$ elemento escolhido para representar este efeito de superfície foi o SURF152, que é um elemento que pode ser aplicado sobreposto em qualquer área de qualquer elemento 3D que requeira uma análise térmica. O elemento pode ser definido por quatro a dez nós, sendo que o mesmo compartilha e se adapta aos nós do elemento sólido adjacente. Um ou dois nós extras (distantes da estrutura principal) podem ser utilizados para aplicar as curvas de incêndio.

\subsection{Curvas de incêndio}

A ação de um incêndio real em uma estrutura é única em cada caso estudado, sendo que jamais podem ocorrer dois incêndios exatamente iguais. $O$ incêndio pode variar devido a inúmeras características da construção em questão, como o material da construção, a existência ou não de aberturas, móveis dentro das compartimentações, etc.

Para facilitar o estudo e definir os procedimentos de ensaios de estruturas em situação de incêndio, curvas nominais padronizadas foram criadas para representar um incêndio independente das características da construção.

A norma brasileira NBR 15200 (ABNT, 2012) informa que a ação em uma estrutura correspondente a um incêndio pode ser representada pela exposição ao incêndio-padrão em um intervalo de tempo (definido na ABNT NBR 14432, de acordo com a ABNT NBR 5628). Este intervalo de tempo é denominado Tempo Requerido de Resistência ao Fogo (TRRF), o qual é definido a partir das características da construção e de seu uso. A curva de incêndio padrão, a qual a norma brasileira se referencia, pode ser calculada pela equação 1, que é fornecida pela norma ISO 834 (2014).

$$
\theta_{g}=\theta_{0}+345 \log (8 t+1)
$$

Na equação 1:

$\mathrm{t}$ : tempo em minutos;

$\theta_{0}$ : temperatura do ambiente antes do início do aquecimento em ${ }^{\circ} \mathrm{C}$;

$\theta_{a}$ : temperatura dos gases no instante $\mathrm{t} \mathrm{em}{ }^{\circ} \mathrm{C}$. 


\subsection{Propriedades termo-mecânicas dos materiais}

Sabe-se que as altas temperaturas alteram o desempenho e as propriedades dos materiais. A resistência de escoamento do aço, assim como a resistência a compressão do concreto são exemplos de propriedades que sofrem decréscimo à medida que a temperatura se eleva. Quando se trata do concreto, a norma NBR 15200 (ABNT, 2012) informa que um fator de redução da resistência deve ser utilizado, fator este que é tabelado de acordo com a temperatura. $\mathrm{O}$ valor da

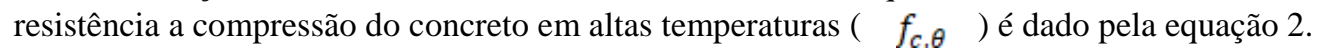

Onde:

$$
f_{c, \theta}=K_{c, \theta} \cdot f_{c k}
$$

$K_{c, \theta}$ : fator de redução da resistência do concreto na temperatura $\theta$;

$f_{c k}$ : resistência característica à compressão do concreto à temperatura ambiente em MPa.

Assim como para o concreto, a resistência ao escoamento da armadura passiva do elemento estrutural também decresce. De forma similar, a norma NBR 15200 (ABNT, 2012) fornece valores tabelados de fatores de redução de resistência ao escoamento do aço para temperaturas elevadas, sendo que os valores se distinguem de acordo com o tipo de aço (CA-50 ou CA-60) e com o tipo de solicitação (tração ou compressão). O módulo de elasticidade do aço é outra propriedade mecânica afetada pelas altas temperaturas, sofrendo decréscimos à medida que a temperatura aumenta.

A equação 3 representa a resistência ao escoamento do aço $\left(f_{v_{0} \theta}\right)$, enquanto na equação 4 tem-se o módulo de elasticidade do aço $\left(E_{S, \theta)}\right)$, ambos para o material aço após exposto a altas temperaturas.

$$
\begin{aligned}
f_{y, \theta} & =K_{S, \theta} \cdot f_{y k} \\
E_{S, \theta} & =K_{E s, \theta} \cdot E_{S}
\end{aligned}
$$

Onde temos:

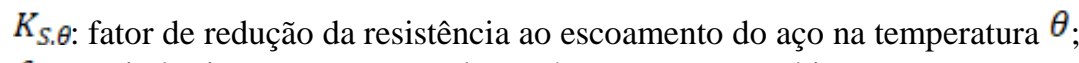

$f_{v, \theta}$ : resistência ao escoamento do aço à temperatura ambiente em MPa;

$K_{E s, \theta}$ : fator de redução do módulo de elasticidade do aço na temperatura $\theta$;

$E_{S:}$ módulo de elasticidade do aço à temperatura ambiente.

Além de determinar a resistência máxima à compressão do concreto e de escoamento do aço a partir do diagrama tensão $\mathrm{x}$ deformação, outra forma de se avaliar a capacidade de uma peça é a partir do momento fletor último da estrutura. Pode-se chegar ao momento último de uma peça com dimensões da seção transversal, armaduras e resistências dos materiais conhecidas a partir de uma verificação à flexão simples onde, por meio de interações, chega-se ao valor de $x$, sendo que este é a distância da linha neutra da seção até a fibra mais comprimida. No mesmo processo pode-se determinar os valores de tensão nas armaduras longitudinais tracionadas $\left(\sigma_{1}\right)$ e comprimidas $\left(\sigma_{2}\right)$, uma vez que a viga em questão é duplamente armada. As equações de equilíbrio da verificação são as apresentadas a seguir como equação 5 (equilíbrio de forças) e equação 6 (equilíbrio de momentos).

$$
\begin{gathered}
\sum F=0 \rightarrow \alpha_{C} \cdot f_{c d^{*}}(b \cdot y)+\sigma_{2} \cdot A_{s}^{\prime}-\sigma_{1} \cdot A_{s}=0 \\
\sum M=0 \rightarrow M_{u}=\alpha_{C} \cdot f_{c d^{*}}(b \cdot y) \cdot(d-0,5 \cdot y)+\sigma_{2} \cdot A_{s^{*}}^{\prime}\left(d-d^{\prime}\right)
\end{gathered}
$$

Onde:

$\alpha_{C}$ : Efeito Rüsh, a ser considerado como 1 devido a carga de incêndio ser instantânea;

$A_{s}$ e $A_{s:}^{\prime}$ área das armaduras inferiores e superiores, respectivamente.

y: altura da linha nêutra em $\mathrm{cm}$.

d: dimensão geométrica, entre o bordo superior (fibra mais comprimida) e o centro da barra de aço tracionada em $\mathrm{cm}$.

d': dimensão geométrica, entre o bordo superior (fibra mais comprimida) e o centro da barra de aço comprimida em $\mathrm{cm}$.

Além da redução da resistência de ambos os materiais, o concreto quando submetido a altas temperaturas sofre, ainda, com a perda de seção resistente. Este fenômeno é conhecido como spalling, e não é diretamente um modo de falha da estrutura, mas sua ocorrêndia pode levar a falhas devido a flambagem das barras de aço e à flexão, por exemplo (FERREIRA, 2019). 
Além dos parâmetros do concreto, há a necessidade de determinar as mesmas propriedades térmicas para o aço, então denominado como material 2, utilizado na armadura longitudinal e transversal da viga. Para tanto, a metodologia utilizada foi semelhante à realizada para o material 1 e os valores estão apresentados nas figuras 4 e 5 a seguir. Os valores de densidade $\left(\rho_{a}(\theta)\right)$ e emissividade $\left(e_{a}\right)$ do aço foram adotados como constantes e iguais a $7850 \mathrm{~kg} / \mathrm{m}^{3}$ e 0,7 , conforme definido nas normas NBR 6118 (ABNT, 2014)

\begin{tabular}{|c|c|c|c|c|}
\hline $\begin{array}{c}\boldsymbol{\theta} \\
{ }^{\circ} \mathrm{C} \\
\end{array}$ & $\begin{array}{c}C_{a} \\
\mathrm{~J} / \mathrm{Kg} .{ }^{\circ} \mathrm{C}\end{array}$ & \multirow{15}{*}{$\begin{array}{l}\left.\text { ! specific heat, J/(kg. }{ }^{\circ} \mathrm{C}\right) \\
\text { MPTEMP, },,,,,, \text { ! Temperatures } \\
\text { MPTEMP, } 1,20 \\
\text { MPTEMP, } 2,100 \\
\text { MPTEMP, } 3,200 \\
\text { MPTEMP, } 4,300 \\
\text { MPTEMP, } 5,400 \\
\text { MPTEMP, } 6,500 \\
\text { MPTEMP, } 7,600 \\
\text { MPTEMP, } 8,700 \\
\text { MPTEMP, } 9,735 \\
\text { MPTEMP, } 10,736 \\
\text { MPTEMP, } 11,800 \\
\text { MPTEMP, } 12,900 \\
\text { MPTEMP, } 13,1000 \\
\text { MPTEMP, } 14,1200\end{array}$} & MPDATA , C , 2, , 439.80 & \multirow[t]{15}{*}{ !values } \\
\hline 20 & 439,80 & & MPDATA, C, $2,, 487.62$ & \\
\hline 100 & 487,62 & & MPDATA,C, $2,, 529.76$ & \\
\hline 200 & 529,76 & & MPDATA, C, $2,, 564.74$ & \\
\hline 300 & 564,74 & & MPDATA,C,2, ,605.88 & \\
\hline 400 & 605,88 & & MPDATA, C, $2,, 666.5$ & \\
\hline 500 & 666,5 & & MPDATA, C, 2, , 759.92 & \\
\hline 600 & 759,92 & & MPDATA, C, 2, , 1008.158 & \\
\hline 700 & 1008,158 & & MPDATA, C, 2, , 5000 & \\
\hline 735 & 5000 & & MPDATA, C, 2, , 4109 & \\
\hline 736 & 4109 & & MPDATA, C, 2, , 803.2609 & \\
\hline 800 & 803,26 & & MPDATA, C, $2,, 650.4438$ & \\
\hline 900 & 650,44 & & MPDATA, C, $2,, 650$ & \\
\hline 1000 & 650 & & MPDATA, C, 2, , 650 & \\
\hline 1200 & 650 & & & \\
\hline
\end{tabular}

Figura 4: Comandos para entrada de dados: calor específico do aço $\mathrm{C}_{\mathrm{a}}$ em função da temperatura

\begin{tabular}{|c|c|c|c|}
\hline $\begin{array}{c}\boldsymbol{\theta} \\
{ }^{\circ} \boldsymbol{C}\end{array}$ & $\begin{array}{c}\boldsymbol{\lambda}_{\mathbf{a}} \\
\mathbf{W} / \mathbf{m}^{\mathbf{2}} \cdot{ }^{\circ} \mathbf{C}\end{array}$ & $\begin{array}{c}\boldsymbol{\theta} \\
{ }^{\circ} \boldsymbol{C}\end{array}$ & $\begin{array}{c}\boldsymbol{\lambda}_{\mathbf{a}} \\
\mathbf{W} / \mathbf{m}^{\mathbf{2}} \cdot{ }^{\circ} \mathbf{C}\end{array}$ \\
\hline 40 & 52,67 & 640 & 32,69 \\
\hline 80 & 51,34 & 680 & 31,36 \\
\hline 120 & 50,00 & 720 & 30,02 \\
\hline 160 & 48,67 & 760 & 28,69 \\
\hline 200 & 47,34 & 800 & 27,30 \\
\hline 240 & 46,01 & 840 & 27,30 \\
\hline 280 & 44,68 & 880 & 27,30 \\
\hline 320 & 43,34 & 920 & 27,30 \\
\hline 360 & 42,01 & 960 & 27,30 \\
\hline 400 & 40,68 & 1000 & 27,30 \\
\hline 440 & 39,35 & 1040 & 27,30 \\
\hline 480 & 38,02 & 1080 & 27,30 \\
\hline 520 & 36,68 & 1120 & 27,30 \\
\hline 560 & 35,35 & 1160 & 27,30 \\
\hline 600 & 34,02 & 1200 & 27,30 \\
\hline
\end{tabular}

\begin{tabular}{|c|c|c|}
\hline \multicolumn{3}{|l|}{ ! thermal conductivity, $\mathrm{W} /\left(\mathrm{cm} .{ }^{\circ} \mathrm{C}\right)$} \\
\hline MPTEMP,,,,,,,,, IT ITemperatures & MPDATA, KXX , 2, ,0.52668 & !Values \\
\hline MPTEMP $, 1,40$ & MPDATA, KXX, 2,, 0.51336 & \\
\hline MPTEMP , 2, 80 & MPDATA, KXX, $2,, 0.50004$ & \\
\hline MPTEMP $, 3,120$ & MPDATA, $K X X, 2,, 0.48672$ & \\
\hline MPTEMP, 4, 160 & MPDATA, KXX, $2,, 0.4734$ & \\
\hline MPTEMP $, 5,200$ & MPDATA, KXX, $2,, 0.46008$ & \\
\hline MPTEMP , 6,240 & MPDATA, $K X X, 2,, 0.44676$ & \\
\hline MPTEMP , 7, 280 & MPDATA $, K X X, 2,, 0.43344$ & \\
\hline MPTEMP , 8, 320 & MPDATA, KXX, $2,, 0.42012$ & \\
\hline MPTEMP, 9,360 & MPDATA, KXX, $2,, 0.4068$ & \\
\hline MPTEMP $, 10,400$ & MPDATA, KXX, 2, ,0.39348 & \\
\hline MPTEMP, 11,440 & MPDATA, $\mathrm{KXX}, 2,, 0.38016$ & \\
\hline MPTEMP $, 12,480$ & MPDATA, $K X X, 2,, 0.36684$ & \\
\hline MPTEMP $, 13,520$ & MPDATA , KXX, 2, , 0.35352 & \\
\hline MPTEMP, 14,560 & MPDATA, KXX, $2,, 0.3402$ & \\
\hline MPTEMP, 15,600 & MPDATA , KXX, 2, , 0.32688 & \\
\hline MPTEMP, 16,640 & MPDATA , KXX, 2, , 0.31356 & \\
\hline MPTEMP, 17,680 & MPDATA , KXX , 2, , 0.30024 & \\
\hline MPTEMP, 18,720 & MPDATA, $\mathrm{KXX}, 2,, 0.28692$ & \\
\hline MPTEMP, 19,760 & MPDATA, KXX, 2, , 0.273 & \\
\hline MPTEMP , 20, 800 & MPDATA, KXX,2, ,0.273 & \\
\hline MPTEMP, 21,1200 & & \\
\hline
\end{tabular}

Figura 5: Comandos para entrada de dados: condutividade térmica do aço $\lambda$ a em função da temperatura

O próximo passo é a determinação da curva de incêndio, para a qual poucas variáveis são necessárias. Os dados utilizados para se traçar a curva são: temperatura inicial $\left(\theta_{0}\right)$ e tempo (t). A NBR 15200 (ABNT, 2012) indica a adoção de uma temperatura inicial de $20{ }^{\circ} \mathrm{C}$.

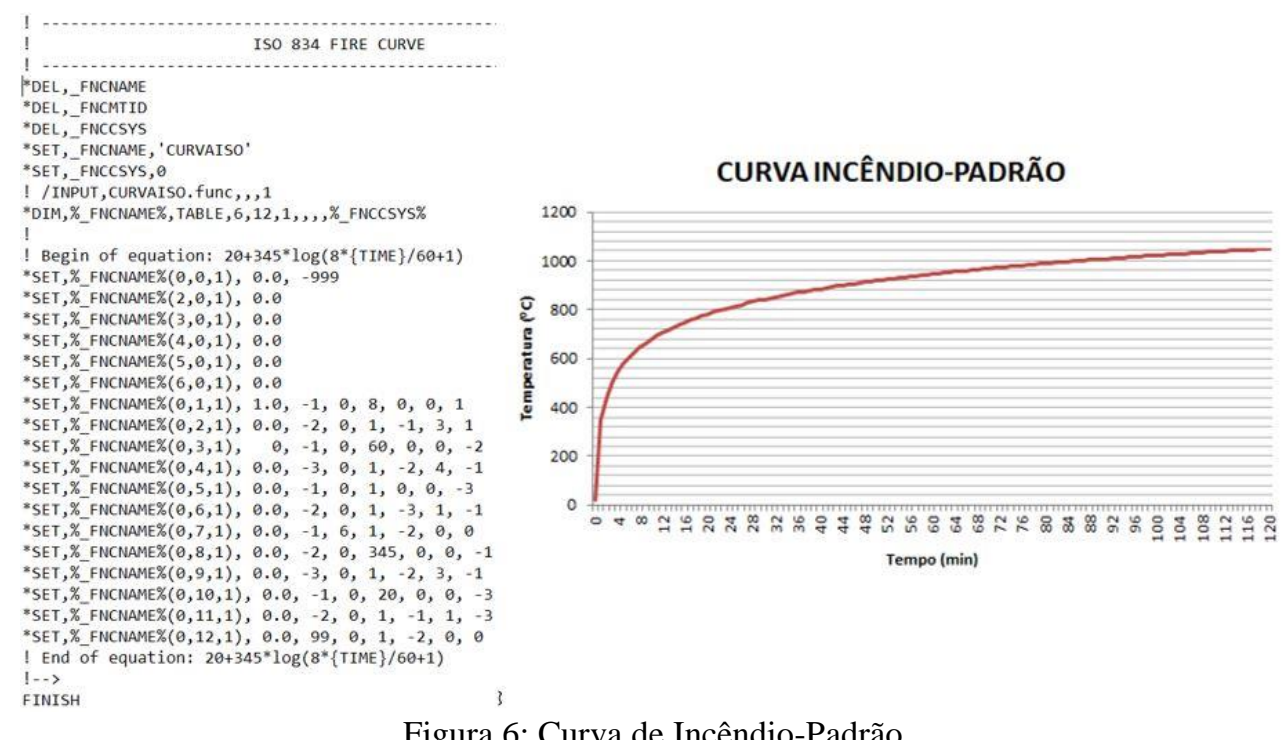

Figura 6: Curva de Incêndio-Padrão 
Assim como as propriedades dos materiais, é necessário informar ao software a variação de temperatura que será aplicada na estrutura. A inserção da curva poderia ser feita por meio de uma tabela, assim como parte das propriedades dos materiais. Todavia, a fim de se obter dados mais exatos a mesma foi definida como uma equação (Figura 6), de forma que o programa possa gerar automaticamente os dados de temperatura para aplicar na estrutura. No decorrer da solução do problema no software, esta curva é aplicada no nó extra, criado pelo elemento SURF152. Vale ressaltar que o nó extra criado pelo elemento SURF152 não representa o foco do incêndio, mas sim um ponto de apoio para que a curva não seja aplicada diretamente na estrutura, a fim de simular os efeitos de convecção e radiação. A variação do tempo se dá por passos de carga que são definidos pelo usuário de acordo com a proposta desejada.

\subsection{Validação do modelo}

A fim de aumentar a credibilidade do modelo computacional realizou-se uma validação do mesmo comparando os resultados obtidos numericamente e os valores obtidos a partir dos ábacos apresentados no anexo A da EN 1992-1-2 (2010). Para tal, modelou-se uma viga de dimensões $\mathrm{h} \mathrm{x} \mathrm{b}=30 \times 16 \mathrm{~cm}$, tal qual a apresentada no Eurocode, usando as propriedades previamente levantadas e os elementos apresentados no item 2.1. Com seção puramente de concreto, a malha aplicada foi de $2 \mathrm{~cm}$ distribuída uniformemente em toda a peça.

Na figura 7 apresentada abaixo, verifica-se a distribuição da temperatura na seção de concreto da estrutura modelada quando submetida ao incêndio representado pela curva de incêndio-padrão pelo período de 30 minutos (a), 60 minutos (b) e 90 minutos (c). Ainda, na figura 8 é possível observar os ábacos de perfis de temperatura consultados, também para os tempos de 30 minutos (a), 60 minutos (b) e 90 minutos (c), os quais constam no EN 1992-1-2 (2010).

Os resultados obtidos por ambos os métodos (software e ábacos) para três pontos distintos da seção são apresentados na tabela 1 . Com um erro médio de $2,98 \%$, pode-se concluir que o modelo desenvolvido é eficiente e pode ser aplicado para outros elementos.

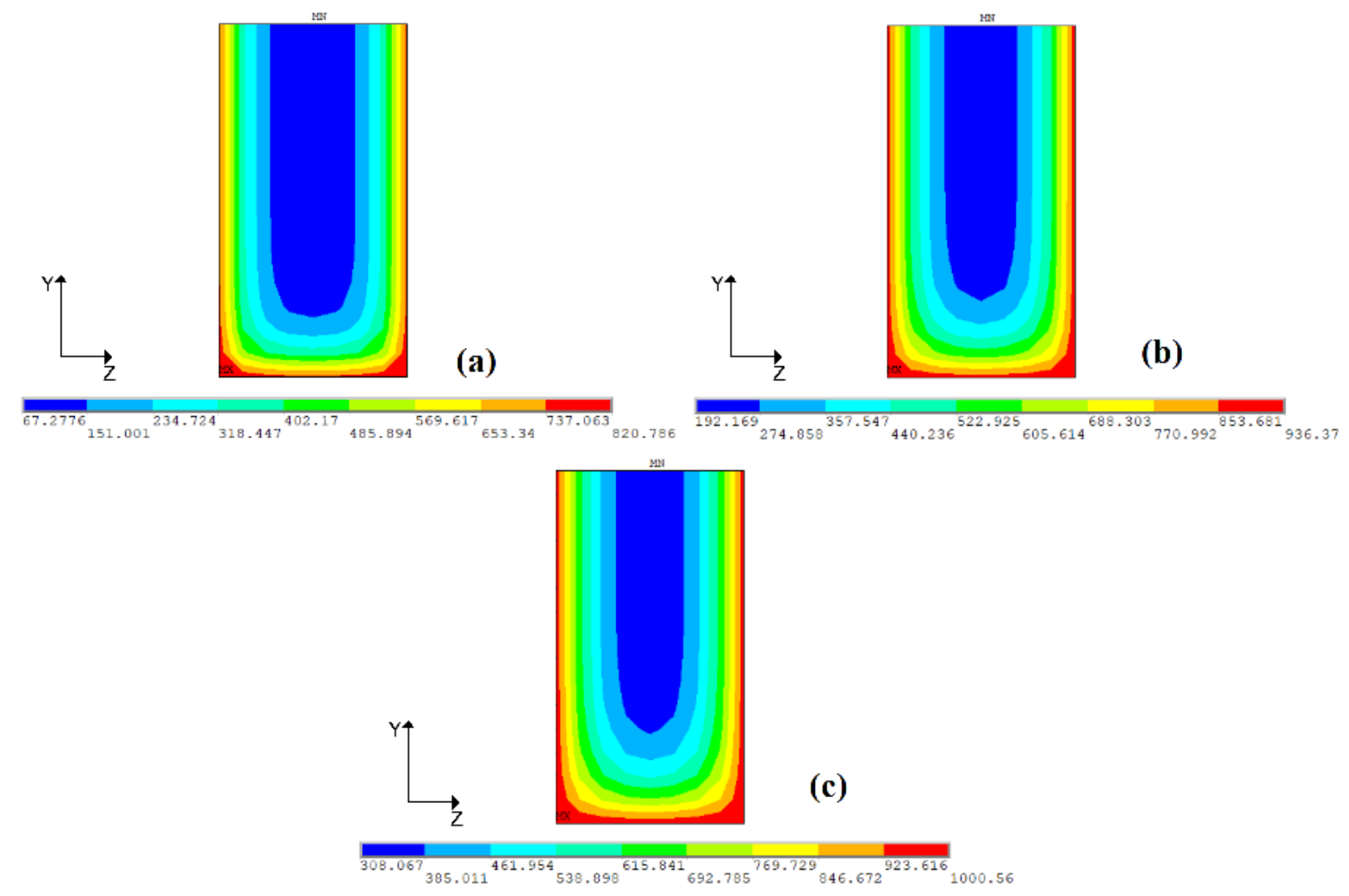

Figura 7: Distribuição da temperatura $\left({ }^{\circ} \mathrm{C}\right)$ na seção de concreto de acordo com o tempo de exposição: (a) 30 minutos; (b) 60 minutos; (c) 90 minutos 


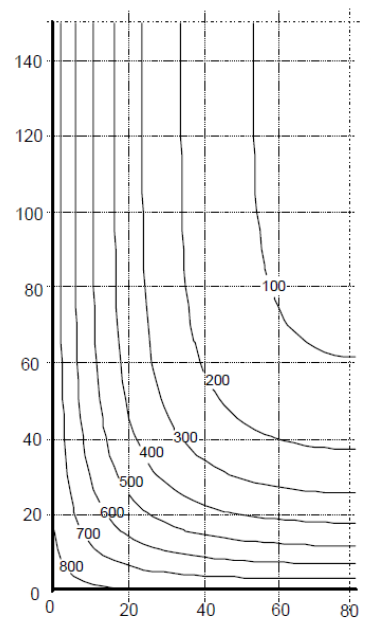

(a) 30 minutos

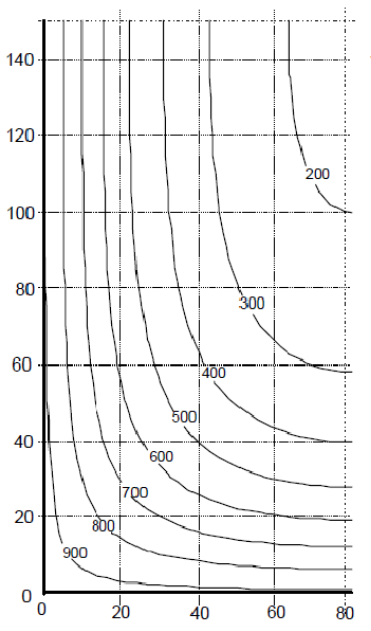

(b) 60 minutos

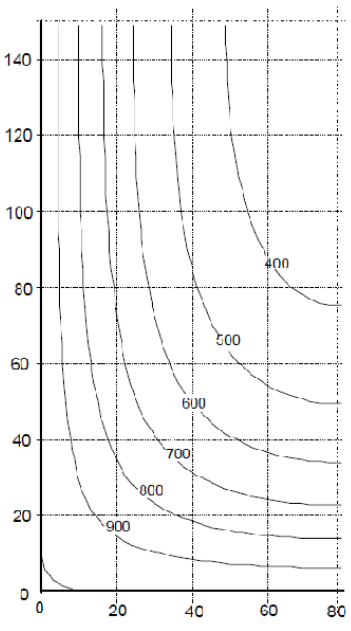

(c) 90 minutos

Figura 8: Perfis de temperatura $\left({ }^{\circ} \mathrm{C}\right)$ para uma viga de $\mathrm{hx} \mathrm{b}=30 \times 16 \mathrm{~cm}$ de acordo com o tempo de exposição: (a) 30 minutos; (b) 60 minutos; (c) 90 minutos

Tabela 1 - Comparação da distribuição de temperatura para uma viga de $\mathrm{h} \times \mathrm{b}=30 \times 16 \mathrm{~cm}$ a partir de um modelo computacional e do Eurocode

\begin{tabular}{c|c|c|c|c|c}
\hline \multirow{2}{*}{$\begin{array}{c}\text { Tempo } \\
(\text { min. }\end{array}$} & \multicolumn{2}{|c|}{$\begin{array}{c}\text { Coordenados dos pontos } \\
\text { analisados }\end{array}$} & \multicolumn{2}{c|}{ Temperatura $\left({ }^{\circ} \mathrm{C}\right)$} & \multirow{2}{*}{$\begin{array}{c}\text { Erro } \\
(\%)\end{array}$} \\
\cline { 2 - 5 } & $\mathrm{Z}(\mathrm{cm})$ & $\mathrm{Y}(\mathrm{cm})$ & ANSYS & Eurocode & \\
\hline 30 & 4 & 2 & 406,65 & 430 & 5,43 \\
\hline 30 & 6 & 4 & 203,82 & 200 & $-1,91$ \\
\hline 30 & 8 & 6 & 115,7 & 120 & 3,58 \\
\hline 60 & 4 & 2 & 628,99 & 650 & 3,23 \\
\hline 60 & 6 & 4 & 404,55 & 420 & 3,67 \\
\hline 60 & 8 & 6 & 288,15 & 290 & 0,63 \\
\hline 90 & 4 & 2 & 760,61 & 780 & 2,47 \\
\hline 90 & 6 & 4 & 551,36 & 570 & 3,27 \\
\hline 90 & 8 & 6 & 430,31 & 460 & 6,45 \\
\hline
\end{tabular}

\subsection{Análise térmica}

O resultado da análise térmica consiste basicamente na determinação das temperaturas em cada nó da malha estrutural da peça, lembrando que, a temperatura se mantém uniforme longitudinalmente enquanto a variação de temperatura se dá na seção transversal da peça (FERREIRA, 2019). A viga analisada, exposta na figura 9, trata-se de uma viga com seção $\mathrm{b} \times \mathrm{h}=30 \times 35 \mathrm{~cm}$, comprimento de $3,0 \mathrm{~m}$, resistência média à compressão de $2,42 \mathrm{kN} / \mathrm{cm}^{2}$ e cobrimento (C) de 2,5 $\mathrm{cm}$. Quanto a armadura utilizada, as mesmas se constituem de 4 barras de $\emptyset 20 \mathrm{~mm}$ e $f_{v}=42,8 \mathrm{kN} / \mathrm{cm}^{2}$ funcionando como armadura inferior, 2 barras de $\varnothing 8 \mathrm{~mm}$ e $f_{v}=46,5 \mathrm{kN} / \mathrm{cm}^{2}$ como armadura superior e estribos de $\varnothing 6 \mathrm{~mm}$ e $f_{v}=32 \mathrm{kN} / \mathrm{cm}^{2}$.

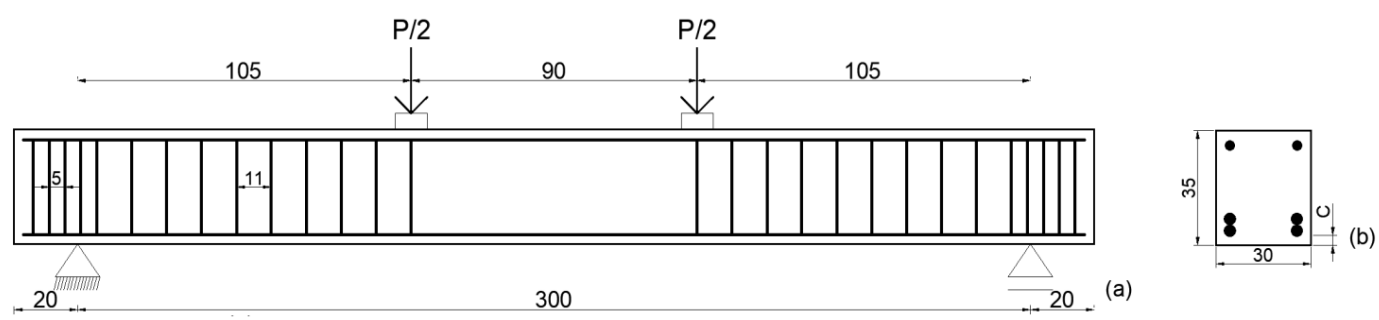

Figura 9: Viga de Leonhadt e Walther: (a) Seção longitudinal; (b) Seção transversal 
A tabela 2 contém as temperaturas do concreto, em ${ }^{\circ} \mathrm{C}$, oriundas da análise térmica da viga em questão para os pontos apresentados na figura 10. As figuras 11 e 12, geradas pelo software ANSYS ao final das análises computacionais mostra o gradiente térmico na seção e na armadura obtido para um tempo de incêndio de 30 minutos (a), 60 minutos (b), 90 minutos (c) e 120 minutos (d). É importante ressaltar que o software ANSYS trabalha com as temperaturas em Kelvin, sendo necessário converter as temperaturas para Celsius. Para tal o comando utilizado é o TOFFST.

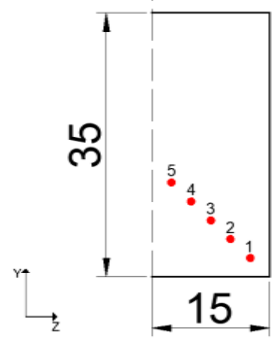

Figura 10: Croqui dos pontos analizados

Tabela 2 - Comparação da distribuição da temperatura de acordo com a variação do cobrimento

\begin{tabular}{|c|c|c|c|c|c|c|}
\hline \multirow{2}{*}{ Nó } & \multicolumn{2}{|c|}{ Coordenados dos pontos analisados } & \multicolumn{4}{|c|}{ Tempo de incêndio (minutos) } \\
\hline & $\mathrm{Z}(\mathrm{cm})$ & $\mathrm{Y}(\mathrm{cm})$ & 30 & 60 & 90 & 120 \\
\hline 1 & 12,5 & 2,5 & 312,87 & 522,41 & 652,01 & 724,94 \\
\hline 2 & 10 & 5 & 160,61 & 333,84 & 460,82 & 557,07 \\
\hline 3 & 7,5 & 7,5 & 73,03 & 188,97 & 290,86 & 376,63 \\
\hline 4 & 5 & 10 & 36,47 & 103,70 & 179,73 & 251,15 \\
\hline 5 & 2,5 & 12,5 & 24,39 & 59,34 & 115,01 & 174,21 \\
\hline \multicolumn{3}{|c|}{ Armadura inferior } & 323,64 & 542,67 & 675,09 & 734,21 \\
\hline \multicolumn{3}{|c|}{ Armadura superior } & 290,27 & 487,70 & 544,72 & 600,93 \\
\hline
\end{tabular}
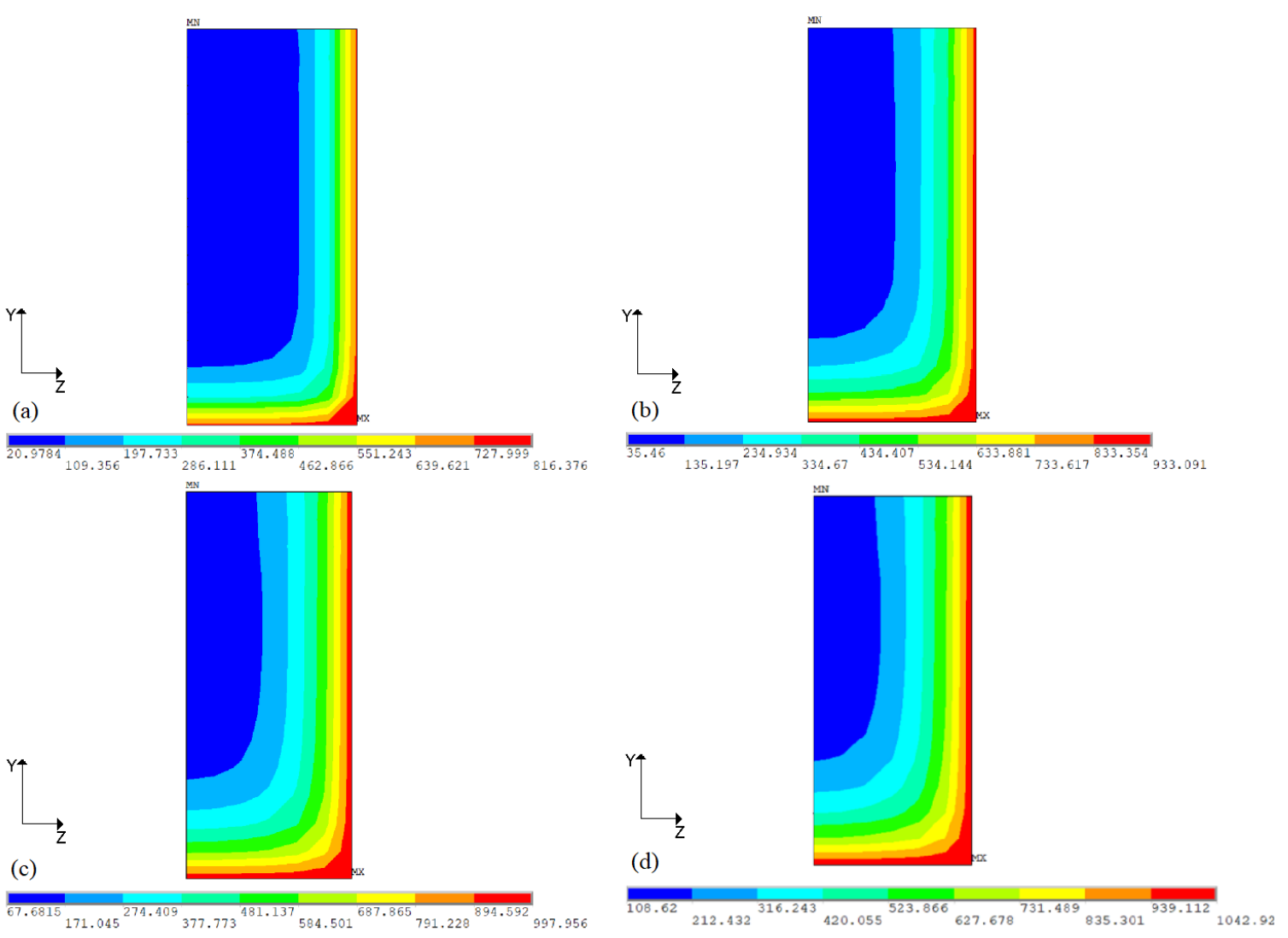

Figura 11: Distribuição de temperaturas $\left({ }^{\circ} \mathrm{C}\right)$ na seção de concreto de acordo com tempo de incêndio: (a) 30 minutos; (b) 60 minutos; (c) 90 minutos; (d) 120 minutos. 

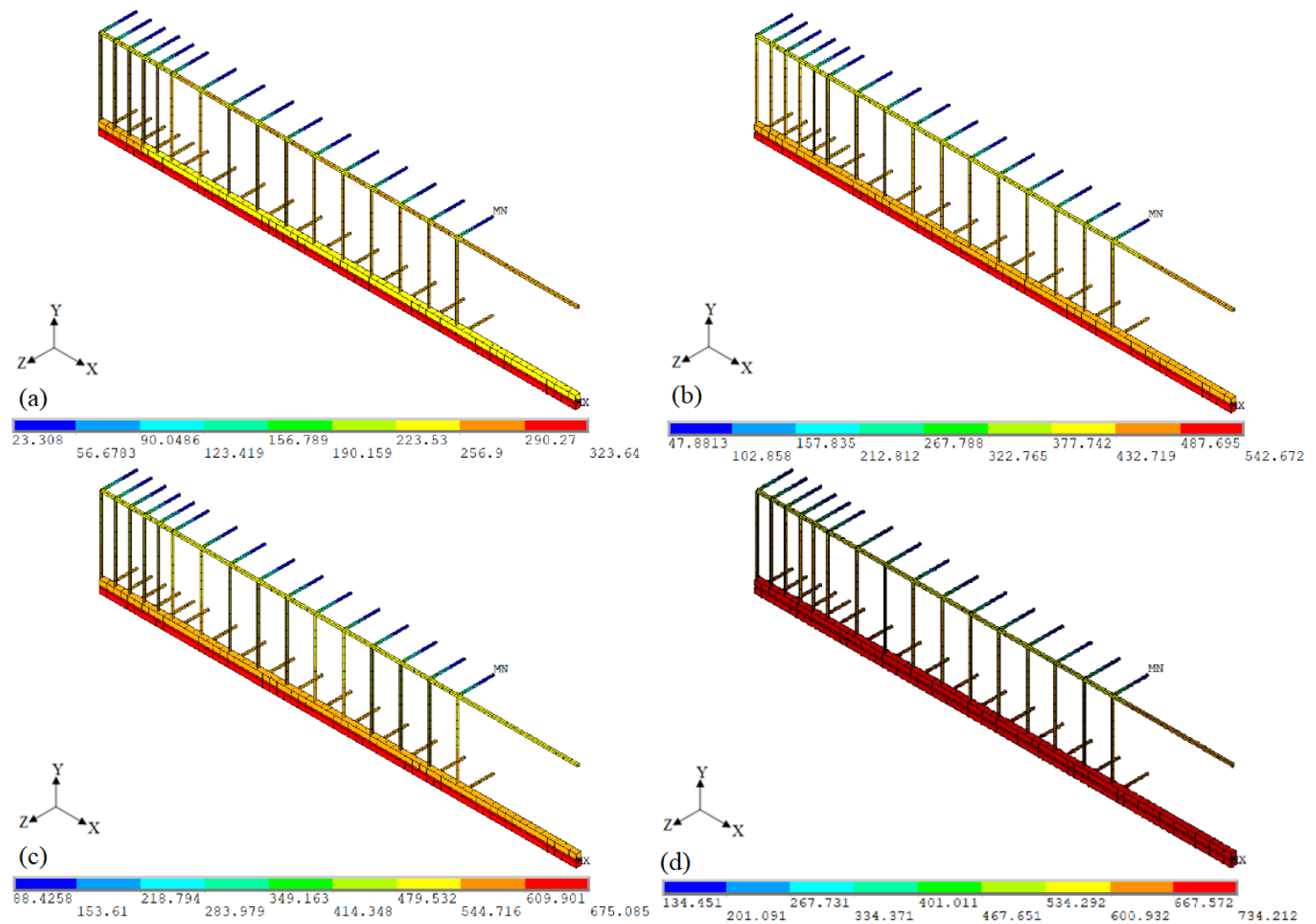

Figura 12: Distribuição de temperaturas $\left({ }^{\circ} \mathrm{C}\right)$ nas armaduras de acordo com tempo de incêndio: (a) 30 minutos; (b) 60

\subsection{Análise estrutural}

minutos; (c) 90 minutos; (d) 120 minutos.

O objeto de estudo deste trabalho é uma viga, que segundo a NBR 6118 (ABNT, 2014), pode ser definida como um elemento linear em que a flexão é preponderante. Assim sendo, foi analisada a variação do momento último resistente à flexão da viga, assim como a redução da resistência característica do concreto $\left(f_{c k}\right)$ e do aço $\left(f_{v k}\right)$, que constitui as armaduras superior e inferior ao longo do incêndio.

Partindo-se inicialmente da viga em temperatura ambiente, foi realizada uma verificação analítica à flexão simples, obtendo-se a localização da linha neutra e o momento último resistente da viga, com o auxílio da equação 6 . Após este processo inicia-se então o tratamento da estrutura em situação de incêndio.

Para averiguar a influência da temperatura no momento resistente último à flexão da viga é necessário primeiramente descobrir os valores da resistência a compressão do concreto em altas temperaturas $\left(f_{c . \theta}\right)$, que é dado pela equação 2 ; bem como da resistência ao escoamento $\left(f_{v \cdot \theta}\right)$ e do módulo de elasticidade $\left(E_{S, \theta}\right)$ do aço em altas temperaturas, que são calculados pelas equações 3 e 4 . A determinação da posição da linha neutra, feita anteriormente, é de extrema importância nesta etapa, uma vez que assim é possível verificar qual é a seção comprimida da viga e determinar a temperatura média no local. O valor da temperatura média na seção comprimida da viga foi encontrada a partir da média ponderada das temperaturas dos pontos do local.

Tendo conhecimento da temperatura média da seção de concreto e da temperatura no aço foi possível determinar os coeficientes de redução de resistência, conforme tabelas da NBR 15200 (ABNT, 2012). Os valores referentes a estes cálculos se encontram na tabela 3. Por fim, determinam-se os momentos últimos à flexão da viga em situação de incêndio. Na tabela 4 foi feita a comparação dos resultados obtidos para a estrutura à temperatura ambiente e em situação de incêndio, com tempos de incêndio de 30 a 120 minutos, os quais serão discutidos no item 4. 
Tabela 3 - Redução da resistência à compressão do concreto e da resistência ao escoamento do aço em altas temperaturas para diferentes tempos de incêndio

\begin{tabular}{|c|c|c|c|c|c|}
\hline \multicolumn{2}{|c|}{$\begin{array}{l}\text { Tempo de incêndio } \\
\text { (minutos) }\end{array}$} & 30 & 60 & 90 & 120 \\
\hline \multirow{3}{*}{$\begin{array}{l}0 \\
0 \\
\stackrel{0}{0} \\
0 \\
0\end{array}$} & $\begin{array}{c}\text { Temperatura } \\
\text { média zona } \\
\text { comprimida }\left({ }^{\circ} \mathrm{C}\right)\end{array}$ & 185,89 & 228,34 & 294,16 & 351,90 \\
\hline & $f_{c k}(\mathrm{MPa})$ & 24,2 & 24,2 & 24,2 & 24,2 \\
\hline & $f_{c, \theta}(\mathrm{MPa})$ & 23,16 & 22,30 & 20,71 & 19,31 \\
\hline \multirow{5}{*}{ 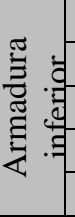 } & Temperatura $\left({ }^{\circ} \mathrm{C}\right)$ & 323,64 & 542,67 & 675,08 & 731,21 \\
\hline & $f_{r k}(\mathrm{MPa})$ & 428 & 428 & 428 & 428 \\
\hline & $f_{\mathrm{r}, \theta}(\mathrm{MPa})$ & 428 & 277,23 & 124,04 & 82,39 \\
\hline & $E_{S}(\mathrm{MPa})$ & 195000 & 195000 & 195000 & 195000 \\
\hline & $\boldsymbol{E}_{S, \theta}(\mathrm{MPa})$ & 151390,20 & 92870,12 & 34095,17 & 22912,50 \\
\hline \multirow{5}{*}{ 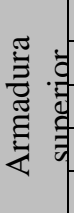 } & Temperatura $\left({ }^{\circ} \mathrm{C}\right)$ & 290,64 & 487,69 & 544,72 & 600,93 \\
\hline & $f_{\mathrm{rk}}(\mathrm{MPa})$ & 465 & 465 & 465 & 465 \\
\hline & $f_{\mathrm{r}, \theta}(\mathrm{MPa})$ & 367,49 & 266,69 & 212,55 & 152,42 \\
\hline & $E_{S}(\mathrm{MPa})$ & 195000 & 195000 & 195000 & 195000 \\
\hline & $E_{S, \theta}(\mathrm{MPa})$ & 157825,20 & 119399,50 & 102493,60 & 60450,00 \\
\hline
\end{tabular}

Tabela 4 - Momentos últimos resistentes a flexão à temperatura ambiente e em altas temperaturas para diferentes tempos de incêndio

\begin{tabular}{c|c}
\hline Tempo de incêndio (mintuos) & $\begin{array}{c}\text { Momento último à flexão } \\
(\mathrm{kN.cm})\end{array}$ \\
\hline 0 (Temperatura ambiente) & 12759,50 \\
\hline 30 & 12575,80 \\
\hline 60 & 8519,35 \\
\hline 90 & 4029,91 \\
\hline 120 & 2716,27 \\
\hline
\end{tabular}

\section{CONSIDERAÇÕES FINAIS}

A proposta inicial deste trabalho era desenvolver uma modelagem computacional a fim de avaliar de forma satisfatória a distribuição térmica em uma viga em situação de incêndio. O processamento numérico dos dados se deu através de uma programação no software ANSYS.

A partir de uma análise térmica, seguida de uma análise estrutural analítica, foi possível visualizar a elevada redução da capacidade da estrutura ao comparar a mesma peça em temperatura ambiente e em situação de incêndio, evidenciando o quanto a estrutura sofre com um sinistro deste tipo. Analisando a tabela 4 foi possível observar que o momento último a flexão da viga decai de $12759,50 \mathrm{kN} . \mathrm{cm}$ para $2716,27 \mathrm{kN} . \mathrm{cm}$, ou seja, uma redução de aproximadamente $78,7 \%$, todavia, temos ciência de que se o efeito spalling fosse considerado, as resistêcias últimas a flexão seriam ainda menores.

Esta perda de resistência tão significativa se dá principalmente pelo aumento da temperatura no aço, uma vez que esta é a parte mais crítica das estruturas quando se encontram em situação de incêndio. Neste cenário, a principal função do concreto se torna proteger passivamente e isolar termicamente o aço da armadura, o que justifica a intenção da ABNT NBR 15200 (2012) ao recomendar, através do Método Tabular, cobrimentos de concreto maiores que os tipicamente utilizados para estruturas projetadas para temperatura ambiente.

Seguindo com as verificações, considerando um coeficiente de segurança $\gamma$ de 1,4 para o momento último à flexão a temperatura ambiente da estrutura, o valor final corresponderia a 9113,92 kN.cm, ou seja, após os 60 minutos de incêndio a peça ja não estaria mais em segurança. De acordo com a NBR 15200 (ABNT, 2012) o cobrimento mínimo para uma viga com $b=300 \mathrm{~mm}$ é $55 \mathrm{~mm}$, enquanto a NBR 6118 (ABNT, 2014) indica um cobrimento mínimo de $25 \mathrm{~mm}$. Estes $25 \mathrm{~mm}$ a mais de espessura de cobrimento para que a estrutura esteja de acordo com a norma de incêndio atualmente vigente poderia vir a garantir a segurança da estrutura e dos usuários durante o TRRF estipulado. 
Conclui-se então que o modelo proposto e desenvolvido é eficiente, assim como o bom desempenho da utilização do software como meio de reduzir custos e tempo em estudos sobre incêndio. Evidencia-se também a importância de seguir as premissas e dimensões mínimas da norma de incêndio NBR 15200 (ABNT,2012) a fim de garantir a segurança da propriedade e da vizinhança, assim como diminuir a perda de vidas humanas.

\section{REFERÊNCIAS}

ASSOCIAÇÃO BRASILEIRA DE NORMAS TÉCNICAS. NBR 14432: exigências de resistência ao fogo de elementos construtivos de edificações - procedimento. Rio de Janeiro, 2001.

NBR 15200: projeto de estruturas de concreto em situação de incêndio. Rio de Janeiro, 2012.

NBR 14323: projeto de estruturas de aço e de estruturas mistas de aço e concreto de edifícios em situação de incêndio. Rio de Janeiro, 2013.

NBR 6118: projeto de estruturas de concreto - procedimento. Rio de Janeiro, 2014.

ANSYS, Inc. Theory reference (Version 2019R1), 2019.

AURICH, M. Modelo da ligação entre concreto e armadura na análise de estruturas de concreto pelo método dos elementos finitos. 2001. 132 f. Dissertação (Mestrado em Engenharia) - Programa de Pós-Graduação em Engenharia Civil, Universidade Federal do Rio Grande do Sul, Porto Alegre, 2001.

CHUMBINHO, H. P. Desempenho estrutural em situação de incêndio - Estabilidade (NBR 15200:2012). Palestra Seminário SINDUSCON - FIEMG, Belo Horizonte, [2017]

EUROPEAN COMMITTEE FOR STANDARDIZATION. EN 1992-1-2: Eurocode 2: design of concrete structures part 1.2: general rules - structural fire design. Brussels: CEN, 2010.

FERREIRA, M. W. Estudo numérico dos efeitos estruturais da exposição de estruturas de concreto armado a altas temperaturas. 2019. 154 f. Dissertação (Mestrado) - Curso de Engenharia Civil, Universidade Federal do Rio Grande do Sul, Porto Alegre, 2019.

FERREIRA, M. W.; SILVA FILHO, L.C.P.; REAL, M. V. Análise numérica termomecânica de estruturas de concreto armado em situação de incêndio. Anais do $61^{\circ}$ Congresso Brasileiro do Concreto, 2019.

INTERNATIONAL ORGANIZATION FOR STANDARDIZATION. ISO 834: Fire resistance tests elements of building construction. Geneva, 2014.

KOCH, H. C. Análise por elementos finitos de vigas em concreto armado através do software ANSYS. $2017.46 \mathrm{f}$. TCC (Graduação) - Curso de Engenharia Civil, Pontifícia Universidade Católica do Rio Grande do Sul, Porto Alegre, 2018.

KUMAR, P.; KODUR, V.K.R. Modeling the behavior of load bearing concrete walls under fire exposure. Construction and Building Materials, Vol. 154, 993-1003, 2017.

LAZZARI, B. M. Análise por elementos finitos de peças de concreto armado e protendido sob estados planos de tensão. 2015. 171 f. Dissertação (Mestrado em Engenharia) - Programa de Pós-Graduação em Engenharia Civil, Universidade Federal do Rio Grande do Sul, Porto Alegre, 2015.

LEONHARDT, F.; WALTHER, R. Beiträge zur behandlung der Schubprobleme im Stahlbetonbau. Beton und Stahlbetonbau. v. 57. n. 7. Jul. 1962.

SORIANO, H. L. Método de elementos finitos em análise de estruturas. São Paulo: Editora da Universidade de São Paulo, 2003. 608 p. Colaboração de Silvio de Souza Lima. 